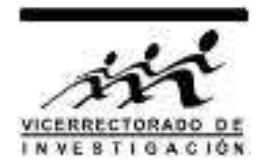

\title{
Análisis de flujo y su influencia en el diámetro medio de las gotas para inyectores de mistura interna tipo $Y$
}

\author{
C. A. Quispe Gonzáles ${ }^{* 1,2}$, W. E. Alvarado Torres $^{2}$, F. S. Costa $^{3}$ y J. A. Carvalho Jr. ${ }^{1}$ \\ ${ }^{1}$ Faculdade de Engenharia de Guaratinguetá, Universidade Estadual Paulista, 12516-41, \\ Guaratinguetá, SP, Brasil \\ ${ }^{2}$ Facultad de Ciencias Físicas, Universidad Nacional Mayor de San Marcos, Lima, Perú \\ ${ }^{3}$ Instituto Nacional de Pesquisas Espaciais, Centro Espacial de Cachoeira Paulista, Brasil
}

Los inyectores de tipo $Y$ son clasificados como inyectores de mistura interna y son muy utilizados en la industria. Las metodologías de cálculo están basadas en las condiciones de flujo sónico al ingresar a la cámara de mistura. En este trabajo, se presenta una metodología de cálculo para estos inyectores en el régimen subsónico donde las condiciones de operación tales como la geometría del inyector, las condiciones de presión y temperatura, las propiedades y gastos másicos del combustible líquido y gasesos de atomización son conocidos. Bajo estas condiciones, un análisis de la ecuación de Wigg -que determina el diámetro medio de las gotas-, muestra que el régimen crítico solo permite obtener el menor diámetro medio de las gotas del spray. Para cualquier otra condición, el gas de atomización fluye bajo un régimen subsónico y el diámetro medio de la gota es mayor que el valor mínimo. Hemos efectuado una comparación entre las ecuaciones que definen el diámetro medio de las gotas en las diferentes variantes de la ecuación de Wigg, propuestas en diferentes trabajos, que obtienen resultados diferentes, esto es debido a una interpretación equivocada de la esencia física de la ecuación de Wigg.

Palabras claves: Inyectores tipo $Y$, diámetro medio de gotas, ecuación de Wigg.

\section{Flux analysis and it's influence on drops mean diameter for $Y$ type internal mixture injectors}

The $Y$ type injectors are classified as internal mixture injectors and are very useful in industries. The calculation methods are based on the sonic flux conditions to enter to mixture chamber. In this work, we present a calculation method for these injectors in the subsonic regime where the operation conditions such as the injector geometry, the pressure and temperature conditions, the properties and mass spend of gaseous and liquid fuel of atomization are known. Under these conditions, an analysis of Wigg equation -which determine the drops mean diameter-, show that, in the critic regime only permit us to obtain the lesser spray drops mean diameter. For any other condition, the atomization gas flows under a subsonic regime and the drop mean diameter is greater that the minimum value. Also, we compare between different equations which define the drop mean diameter in differents variants of Wigg equations, propossed in different published woks, which obtain different results, this is due to a wrong interpretation of the physical essence of the Wigg equation.

Keywords: $Y$ type injector, drops mean diamter, Wigg equation.

\footnotetext{
*cquispeg@yahoo.com
} 
La atomización del combustible es la desintegración del líquido en pequeñas gotas antes de su inyección a la zona de combustión. Este proceso es fundamental para la eficiencia de la combustión y el desempeño de un horno industrial, ya que ayuda a promover la vaporización del combustible y la transferencia de calor al generar una alta razón de superficie-volumen en la fase líquida. Un buen proceso de atomización debe tener la capacidad de asegurar la energía suficiente para la atomización con una buena eficiencia y un bajo costo, en una amplia faja del gasto de combustible líquido, así como responder efectivamente a las variaciones en el caudal del combustible líquido [1].

Los inyectores tipo $Y$ son clasificados como inyectores de chorro de aire y de mezcla interna. Son muy utilizados debido a: 1) configuración relativamente simple, 2) adaptabilidad de ser construido con materiales resistentes a la erosión y corrosión térmica, 3) ángulo del spray estable al variar el caudal de combustible, 4) spray de buena calidad con bajo consumo de gas de atomización y 5 ) capacidad de trabajo con combustibles altamente viscosos. Estos inyectores, inicialmente desintegran el combustible líquido dentro de una cámara de mistura, generando gotas y una capa fina que se adhiere a la pared de la cámara, prosiguiendo la quiebra de esta capa fuera del inyector. El combustible, ya desintegrado en finas gotas, se dispersa dentro de la cámara de combustión, donde ocurre la quema de estas gotas.

Muchos investigadores han realizado estudios sobre la estructura del fluido atomizado y la caracterización del spray generado por este inyector [2-11]. El efecto de la geometría del inyector sobre el diámetro medio de las gotas fue analizado en [12], proponiendo una metodología para el cálculo y diseño de este tipo de inyectores, utilizada luego en [13]. Utilizando los datos obtenidos por [14]. Se presentan ecuaciones para el cálculo del diámetro medio de las gotas [14], Wigg consiguió desarrollar una relación empírica para predecir el diámetro medio de las gotas del spray [15] conocida actualmente como ecuación de Wigg y que da buenos resultados para datos de atomización utilizando aire o vapor como gas de atomización y combustibles líquidos viscosos [12] pero da resultados discrepantes cuando se usa aireagua $[16,17]$. Otros investigadores $[18,19]$ presentan ecuaciones similares para el diámetro medio, basadas en la Ecuación de Wigg.

La mayoría de los investigadores admite que las propiedades del gas de atomización a la salida de la tobera, antes de ingresar a la cámara de mezcla, deben tener condiciones críticas. En este trabajo, se asume las condiciones críticas en la etapa de cálculo y diseño del inyector, pero para cualquier otra condición, el flujo de gas de atomización se encuentra en el régimen subsónico y genera una atomización con moderada calidad, donde el diámetro medio de la gota puede alcanzar medidas menores de $80 \mu \mathrm{m}$.

\section{Teoría}

La ecuación de Bernoulli para líquidos y gases puede ser derivada de la ecuación de Euler [20], de la ecuación de Navier-Stokes [21] o de los principios termodinámicos [22] y describe los flujos ideales a través de difusores, ductos e inyectores, en los cuales el fluido fluye bajo condiciones adiabáticas sin realizar trabajo. Esta ecuación está dada por

$$
\frac{V^{2}}{2}+\int \frac{d P}{\rho}+g z=\text { constante } .
$$

Esta ecuación también puede ser usada para describir el comportamiento de fluidos a la entrada de la cámara de mezcla de inyectores tipo $Y$. El término que representa la energía potencial puede ser omitido en comparación con los otros términos debido a la pequeña escala del atomizador. Para el caso de la línea de inyección del combustible líquido, la densidad es considerada constante y la ecuación (1) puede reescribirse como

$$
\frac{V^{2}}{2}+\frac{P}{\rho}
$$

Dentro del reservorio o tanque de combustible, la velocidad es nula, la presión es igual a la presión de estancamiento que es igual a la presión de inyección del combustible $P_{F}$. De hecho, el valor de la presión que existe en este reservorio es una diferencial total de presión e igual a la presión manométrica in gauge encima de la presión atmosférica. Si la presión en la cámara de mezcla es $P_{M}$ entonces se puede definir la diferencial de presión $\Delta P$ necesaria para impulsar el combustible desde el tanque hasta la cámara del inyector. De la ecuación (2) se obtiene

$$
\frac{P_{f}}{\rho}=\frac{V_{C}^{2}}{2}+\frac{P_{M}}{\rho} \Rightarrow \frac{\Delta P}{\rho}=\frac{P_{F}-P_{M}}{\rho}=\frac{V_{C}^{2}}{2},
$$

donde $V_{C}$ es la velocidad con la cual el combustible ingresa a la cámara de mezcla del inyector. 
De manera similar, para la línea de inyección del gas de atomización, la integral de la ecuación (1) es calculada considerando un flujo adiabático e isoentrópico. Calculando la integral y despreciando el término de energía potencial $\rho g$ se obtiene [22]

$$
\frac{V^{2}}{2}+\frac{k}{k-1} \frac{P}{\rho}=\text { constante }
$$

donde $k=C_{p} / C_{v}$ es la razón de calores específicos del gas.

La Figura 1 muestra un esquema simplificado de la línea de abastecimiento de gas comprimido y del inyector, donde el reservorio, presurizado por un compresor o por la presión de algún gas, tiene un manómetro M1 que mide la presión manométrica in gauge y es igual a la presión de estancamiento $P_{0}$. La válvula reguladora $\mathrm{V}$ permite variar el gasto másico del gas a través de la línea, que a su vez varía la razón gas-combustible, gas-liquid ratio (GLR). Así, la presión medida con el manómetro M2 es igual a la presión estática del gas $P_{V}$ aguas debajo de la válvula. El manómetro M3 mide la presión de inyección $P_{I}$ en la tobera del inyector, a la entrada de la cámara de mezcla. El inyector puede ser considerado como una tobera convergente, donde $P_{I}$ es la presión en la línea de inyección y $P_{S A L}$ es la presión a la salida de la tobera, que es la entrada a la cámara del inyector. La presión estática $P_{M}$ representa la presión en la cámara de mezcla y $P_{A T}$ la presión fuera del inyector, igual a la presión atmosférica. La temperatura de estancamiento $T_{o}$ es dada por la temperatura ambiente.

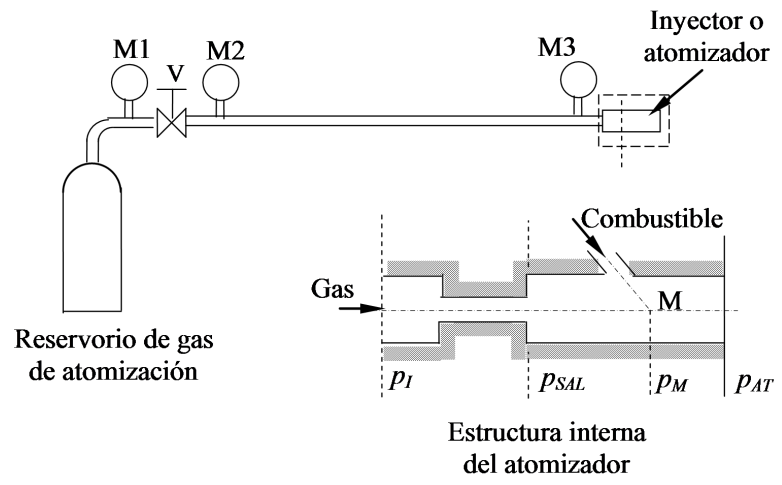

Figura 1: Esquema de la línea de gas de atomización y esquema del inyector tipo $Y$.

La presencia de la válvula origina una caída de presión $\Delta P_{V}$ en la línea de inyección de gas. Enton- ces, de acuerdo con la ecuación (4) obtenemos

$$
\begin{gathered}
\frac{\gamma}{\gamma-1} \frac{P_{0}}{\rho_{0}}=\frac{V_{V}^{2}}{2}+\frac{\gamma}{\gamma-1} \frac{P_{V}}{\rho_{V}}+\frac{\gamma}{\gamma-1} \frac{\Delta P_{V}}{\rho_{V}} \Rightarrow \\
\frac{\gamma}{\gamma-1}\left(\frac{P_{0}}{\rho_{0}}-\frac{\Delta P_{V}}{\rho_{V}}\right)=\frac{V_{V}^{2}}{2}+\frac{\gamma}{\gamma-1} \frac{P_{V}}{\rho_{V}} .
\end{gathered}
$$

Los parámetros de estancamiento en el reservorio pueden ser conocidos por medición directa, pero la estimación de la caída de presión en la válvula reguladora es más compleja, debido a que al variar el gasto másico del gas -abriendo o cerrando la válvula-, también variará la caída de presión. De otro lado, es mejor estimar las magnitudes del lado derecho de la ecuación (5), aguas debajo de la válvula y que obedecen a la ecuación (4). La presión $P$ puede ser medida con un manómetro, mientras que la densidad $\rho$ puede ser estimada con ayuda de la ecuación de estado si se conoce la temperatura $T$, la misma que puede ser medida con un termómetro o termopar mediante

$$
\rho=\frac{P}{R T} .
$$

La medición directa de la velocidad $V$ ofrece mayor dificultad. Si el diámetro del conducto fuera lo suficientemente grande se podría instalar un tubo de Pitot y medir la presión dinámica. Generalmente, en este tipo de instalaciones, los conductos son de diámetro pequeño, por lo que la estimación de la velocidad $V$ debe ser efectuada de forma indirecta, a través del gasto másico. Instalando un medidor de gasto, rotámetro o medidor digital, se puede conocer el caudal volumétrico del gas $Q_{g}$. Además, siendo conocido el diámetro del tubo de gas $d_{g}$, se conoce el área de la sección transversal, por lo que la velocidad media puede ser estimada como

$$
V=\frac{4 Q_{g}}{\pi d_{g}^{2}}
$$

Cada nueva variación de la válvula reguladora produce una variación en los parámetros del flujo aguas debajo de ella. Los parámetros de estancamiento aguas debajo de la válvula son dados por

$$
\frac{\gamma}{\gamma-1} \frac{P_{o}^{\prime}}{\rho_{o}^{\prime}}=\frac{V^{2}}{2}+\frac{\gamma}{\gamma-1} \frac{P}{\rho}=\text { constante } .
$$

Estos parámetros de estancamiento, idealmente, solo son iguales a los parámetros de estancamiento 
del tanque si la válvula está totalmente abierta. Para cualquier otro caso, estos parámetros de estancamiento son menores que los del reservorio. La temperatura de estancamiento permanece constante para cualquier caso.

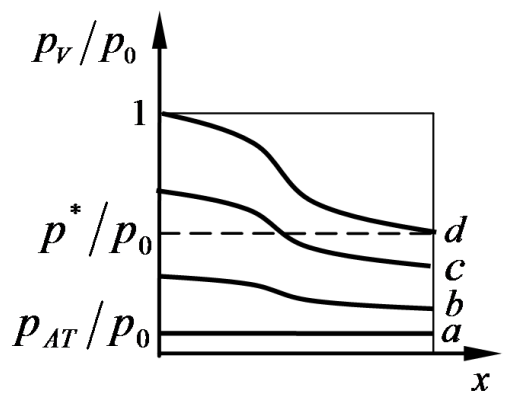

a)

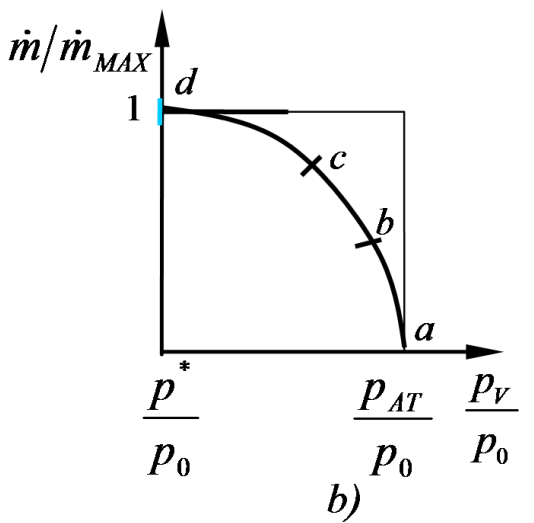

Figura 2: Variación en la presión: a) aguas debajo de la válvula, b) variación del gasto másico.

Si la válvula $\mathrm{V}$ está totalmente abierta, la presión de estancamiento en toda la línea es igual a la del tanque $P_{0}^{\prime}=P_{0}$, son conocidos, también, la geometría del inyector, las propiedades físicas del gas de atomización como la constante del gas $R$ y la razón de calores específicos $k$. Por tanto, el gasto másico máximo del gas a través del inyector conociendo los parámetros de estancamiento $P_{0}$ y $T_{0}$ es calculado por

$$
\dot{M}_{\max }=\dot{m}^{*}=\frac{A P_{0}}{\sqrt{R T_{0}}} k^{\frac{1}{2}}\left(\frac{2}{k+1}\right)^{\frac{k+1}{2(k-1)}} .
$$

En este caso, los parámetros termodinámicos son críticos, $P^{*}, T^{*}, \rho^{*}, V^{*}=a^{*}$, siendo $a$ la velocidad local del sonido. Si la válvula está totalmente cerrada no habrá ningún flujo por el inyector, la velocidad es nula y el gasto másico es cero, línea $a$ de la Figura 2.a. Abriendo poco a poco la válvula, el gas comienza a fluir y la presión $P_{V}$ medida por el manómetro M2 aumenta, estableciendo nuevos parámetros de estancamiento en la línea de inyección $P_{0}^{\prime}>P_{V}$, ocasionando un incremento en el gasto másico, líneas b, c y d de la Figura 2.b.

Este incremento continua hasta que la relación de presión $P_{V} / P_{0}^{\prime}$ es igual a la relación crítica $P^{*} / P_{0}^{\prime}$, luego el gasto másico alcanza el valor máximo o crítico. Para las relaciones de presión mayores que la relación crítica $P_{\mathrm{SAL}} / P_{0}^{\prime}>P^{*} / P_{0}^{\prime}$ el flujo a través del inyector se encuentra en un régimen subsónico. Se debe indicar también, que este proceso ocurre en la válvula, siempre que la sección de paso a través de ella sea menor que la sección de paso de la tobera del inyector. Cuando ocurre el caso inverso, la sección crítica se traslada a la salida de la tobera, entrada a la cámara de mezcla.

Como el interés es el análisis del proceso dentro del inyector, es conveniente considerar que el fenómeno de bloqueo ocurre a la salida de la tobera del inyector y en condiciones críticas, donde los parámetros son [22]

$$
\begin{aligned}
\frac{P_{\mathrm{SAL}}}{P_{0}^{\prime}} & =\frac{P^{*}}{P_{0}^{\prime}}=\left(\frac{2}{k+1}\right)^{\frac{k}{k-1}} \\
\frac{T_{\mathrm{SAL}}}{T_{0}^{\prime}} & =\frac{T^{*}}{T_{0}}=\frac{2}{k+1} \\
\rho_{\mathrm{SAL}} & =\rho^{*}=\frac{P_{\mathrm{SAL}}}{R T_{\mathrm{SAL}}} .
\end{aligned}
$$

Los resultados obtenidos por [22] muestran que las condiciones de bloqueo no dependen de la presión fuera del inyector, sino de los parámetros del flujo gaseoso dentro de la cámara de mezcla y de las condiciones de estancamiento en la línea de inyección de gas, influenciados a su vez por la presión de inyección del combustible. Por lo tanto, es importante determinar los parámetros de estancamiento en la línea de flujo gaseoso.

Dado que la determinación de los parámetros del flujo gaseoso ofrece una serie de dificultades, es necesario tener los datos de entrada, a partir de los cuales se puedan calcular los demás parámetros del flujo a la salida de la tobera del inyector. Entre estos parámetros se tiene a la presión de inyección de combustible, que se puede asumir igual a la presión de salida $P_{\mathrm{SAL}}$ y el gasto másico de combustible $\dot{m}_{F}$ puede ser establecido de antemano, por el manipuleo de las válvulas de la bancada de experimentación. Así también, debe 
definirse la relación GLR, que de acuerdo a las recomendaciones dadas por [12], se encuentra en el intervalo $0.05-0.15$. La temperatura de estancamiento $T_{0}$ es igual a la temperatura ambiente y permanece constante en todo el proceso. Entre las características geométricas del inyector debe conocerse el diámetro del conducto de gas $d_{g}$. Las propiedades del gas de atomización son conocidas, $R$ y $k$.

Para las condiciones de bloqueo, la presión de estancamiento y temperatura del gas a la salida de la tobera son

$$
\begin{aligned}
& \frac{P^{*}}{P_{0, g}^{\prime}}=\frac{P_{\mathrm{SAL}}}{P_{0, g}^{\prime}}=\left(\frac{2}{k+1}\right)^{\frac{k}{k-1}} \\
& \frac{T^{*}}{T_{0, g}^{\prime}}=\frac{T_{\mathrm{SAL}}}{T_{0, g}}=\frac{2}{k+1} .
\end{aligned}
$$

A su vez, la densidad del gas a la salida de la tobera y la densidad de estancamiento son

$$
\rho_{\mathrm{SAL}}=\rho^{*}=\frac{P_{\mathrm{SAL}}}{R T_{\mathrm{SAL}}} \quad \rho_{0, g}=\frac{P_{0, g}}{R T_{0, g}} .
$$

Conocidos $P_{0}$ y $T_{0}$, así como las propiedades del gas, $k$ y $R$; y como $A=\pi d_{g}^{2} / 4$, entonces es posible calcular el gasto másico máximo de gas a través del inyector mediante la ecuación (9). Si el gasto másico de gas dado es igual al gasto máximo, entones el flujo se encontrará en condiciones críticas, caso contrario, se encontrará en condiciones de régimen subsónico. Para calcular los parámetros en régimen subsónico se sigue el siguiente procedimiento, $a$ ) se define el gasto específico como la razón entre el gasto másico y el gasto másico máximo,

$$
q=\frac{\dot{m}}{\dot{m}_{\max }}=\frac{\dot{m}}{\dot{m}^{*}}=\frac{\rho A V}{\rho^{*} A^{*} V^{*}}=\frac{\rho}{\rho_{0}} \frac{\rho_{0}}{\rho^{*}} \frac{V}{a} \frac{a}{a_{0}} \frac{a_{0}}{a^{*}} ;
$$

b) las relaciones de densidad para el flujo adiabático e isoentrópico [22], se establecen como

$$
\begin{aligned}
\frac{\rho_{0}}{\rho} & =\left(1+\frac{k-1}{2} M^{2}\right)^{\frac{1}{k-1}}, \\
\frac{\rho^{*}}{\rho_{0}} & =\left(\frac{2}{k+1}\right)^{\frac{1}{k-1}} ;
\end{aligned}
$$

c) para las relaciones de velocidades, tenemos

$$
\begin{aligned}
M & =\frac{V}{a}, \\
\frac{a_{0}}{a} & =\left(1+\frac{k-1}{2} M^{2}\right)^{\frac{1}{k-1}}, \\
\frac{a^{*}}{a_{0}} & =\left(\frac{2}{k+1}\right)^{\frac{1}{2}} ;
\end{aligned}
$$

d) sustituyendo las relaciones de las ecuaciones (14) y (15) en la ecuación (13) se puede obtener

$$
q=\frac{\dot{m}}{m^{*}}=M\left[\frac{2+(k+1) M^{2}}{k+1}\right]^{-\frac{k+1}{2(k-1)}} .
$$

La ecuación (14) proporciona dos soluciones, la primera para el régimen subsónico con $M<1$ y la segunda para el régimen supersónico con $M>1$, siendo los valores buscados menores a la unidad. Determinado el número de Mach a la salida de la tobera, se encuentra la velocidad del flujo y la velocidad local del sonido con ayuda de la ecuación (15).

La presión $P_{M}$ en la cámara de mezcla del inyector es diferente a la presión de salida de la tobera. El chorro al salir de la tobera pasa por una expansión brusca y se expande, provocando una caída de la presión y la consecuente variación de la velocidad y de la densidad del flujo. La geometría de la cámara de mezcla juega un papel muy importante en este caso. La pérdida de presión por expansión brusca localizada puede ser estimada por medio de

$$
\begin{aligned}
& \Delta P=K_{\text {perd }} \frac{\rho_{\mathrm{SAL}} V_{\mathrm{SAL}}^{2}}{2}, \\
& P_{M}=\left(1-k_{\mathrm{perd}}\right) P_{\mathrm{SAL}},
\end{aligned}
$$

donde $K_{\text {perd }}$ es el coeficiente de pérdida de la presión, el cual debe ser determinado experimentalmente para cada situación. El coeficiente $K_{\text {perd }}$ puede ser determinado mediante la expresión [24]

$$
K_{\text {perd }}=\left[1-\left(\frac{d_{\mathrm{SAL}}}{d_{M}}\right)^{2}\right]^{2} .
$$

El trabajo realizado por [2] caracterizó dos tipos de inyectores tipo $Y$, así también midió la caída de presión a través del inyector, Fig. 3.a, donde el punto P1 se encuentra cerca del punto de mezcla. Estos datos pueden ser utilizados para obtener la pérdida de presión desde la salida de la tobera hasta la cámara de mezcla. Los datos de la Fig.4.a fueron llevados a coordenadas adimensionales y se muestran en la Fig.3.b. 


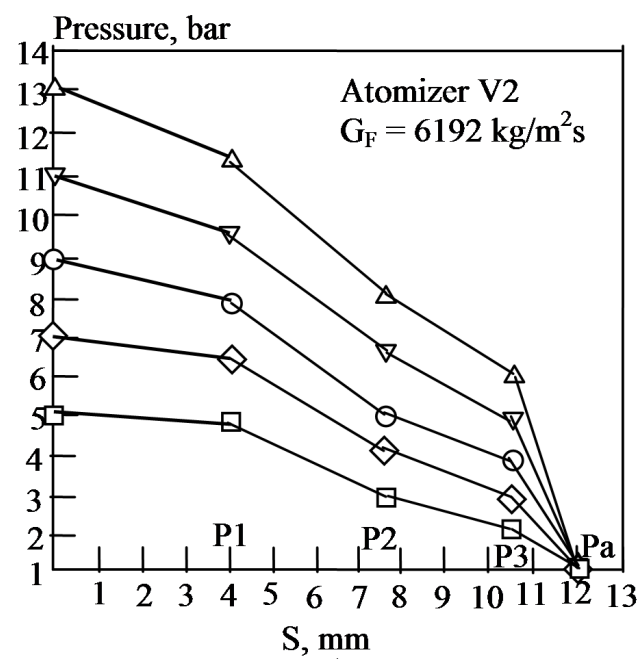

a)

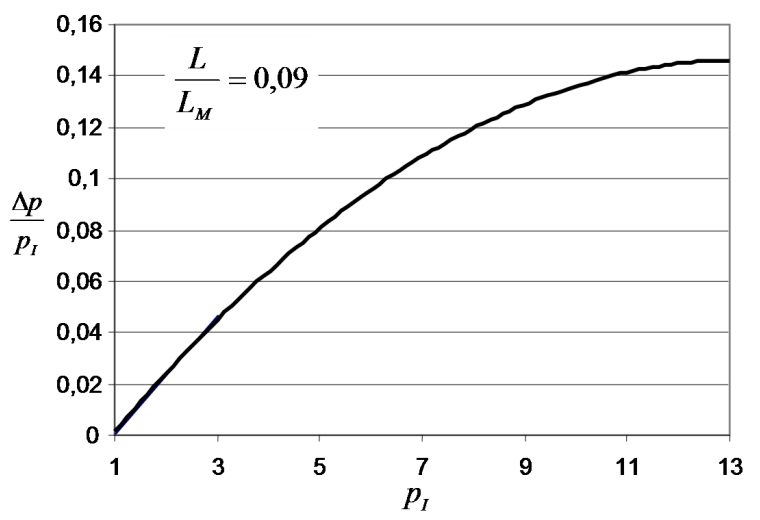

b)

Figura 3: Pérdida de presión: a) a través del inyector [2], b) pérdida adimensional a través del inyector.

A pesar de existir pocos datos, se observa de la Fig.3.a y 3.b que la caída de presión entre la salida de la tobera y el punto de la mezcla obedece a una ley cuadrada y que concuerda con los conceptos establecidos por la mecánica de fluidos para expansiones bruscas. Esto permite aceptar la validez de la Fig.3.b. La presión en el punto de mezcla es

$$
P_{M}=P_{I}-\Delta P \text {. }
$$

Conocida la presión en la cámara de la mezcla, la densidad es determinada por

$$
\frac{\rho_{M}}{\rho_{S A L}}=\left(\frac{P_{M}}{P_{\mathrm{SAL}}}\right)^{\frac{1}{k}} .
$$

La caída de la presión entre la salida de la tobera $P_{\mathrm{SAL}}$ y la salida del inyector $P_{A T}$ se debe a la influencia de la expansión brusca, la colisión directa del chorro líquido y el chorro de gas, la quiebra primaria del chorro líquido, la generación de gotas, la formación de la capa de líquido en la pared de la cámara de la mezcla, la quiebra secundaria del chorro fuera del inyector generando gotas más pequeñas, además de otros fenómenos asociados al flujo bifásico. Obtenidos los parámetros del flujo de gas de atomización en la cámara de mezcla, es posible determinar los parámetros en la línea de inyección del combustible líquido. De acuerdo a recomendaciones [12], el diámetro del conducto de inyección del combustible es igual al diámetro del conducto de gas, $d_{F}=d_{g}$. El coeficiente de descarga $C_{D}$ se define como la razón entre el gasto másico real y el gasto másico teórico,

$$
C_{D}=\frac{\dot{m}_{F_{\mathrm{REAL}}}}{\dot{m}_{F_{\mathrm{TEOR}}}} .
$$

Para determinar el gasto másico de combustible líquido es necesario conocer la velocidad del líquido con la cual llega a la cámara de mistura. La ecuación (3) permite determinar

$$
V_{F}=\sqrt{\frac{2 \Delta P_{F}}{\rho_{F}}},
$$

donde $\rho_{F}$ es la densidad del combustible, $\Delta P_{F}=$ $P_{F}-P_{M}$ es la caída de la presión que existe desde el tanque de combustible hasta la cámara de la mezcla e igual a la diferencia de la presión en el tanque y $P_{F}$ la presión en la cámara de la mezcla $P_{M}$.

La siguiente expresión empírica permite predecir el tamaño medio de las gotas producidas por el inyector $Y$

$$
D_{0.5}=\frac{200 V^{0.5} \dot{m}_{F}^{0.1}\left(1+\dot{m}_{F} / \dot{m}_{A}\right)^{0.5} h^{0.1} \sigma^{0.2}}{\rho_{A T}^{0.3} \Delta U}
$$

donde $D_{0.5}$ es el diámetro medio de la masa de las gotas del spray, en $\mu \mathrm{m} ; V$ es la viscosidad cinemática del combustible, en cSt; $\dot{m}_{F}$ es el gasto másico de combustible, en $\mathrm{g} / \mathrm{s} ; \dot{m}_{A}$ es el gasto másico de gas de atomización, en $\mathrm{g} / \mathrm{s} ; h$ es la altura del anillo de aire, en $\mathrm{cm} ; \sigma$ es la tensión superficial del combustible, en dinas $/ \mathrm{cm}, \sigma_{A T}$ es la densidad del gas en el punto de mezcla, en $\mathrm{g} / \mathrm{cm}^{3} ;$ y $\Delta U$ es la velocidad del gas de atomización relativo a la velocidad del combustible, en $\mathrm{m} / \mathrm{s}$. El análisis de esta ecuación muestra que la velocidad relativa $\Delta U$ es importante, pues 
cuanto mayor sea, menor será el diámetro de las gotas aunque también es afectada por la razón másica líquido/gas. Esto también explica que las fuerzas de cizallamiento predominen sobre las fuerzas de tensión superficial. La geometría del atomizador tiene una leve influencia ya que la película líquida que se forma en la pared de la cámara se ve levemente influenciada por la geometría del inyector que tiene exponente de 0.1. Si bien es cierto que la calidad de la atomización está dada por el tamaño de las gotas, se pueden generar gotas de diámetro razonable, menores a $80 \mu \mathrm{m}$, lo cual es posible conseguir eligiendo convenientemente la presión de estancamiento $P_{o}^{\prime}$. Para las condiciones críticas, el diámetro de las gotas alcanza su valor minimo.

Otros investigadores $[1,18]$ transformaron la ecuación de Wigg al Sistema Internacional, con $D_{0.5}$ en $\mathrm{m}$, en $\mathrm{m}^{2} / \mathrm{s}, \dot{m}_{F}$ y $\dot{m}_{A}$ en $\mathrm{kg} / \mathrm{s}, \mathrm{h}$ en $\mathrm{m}, \sigma$ en $\mathrm{N} / \mathrm{m}$, $\sigma_{A T}$ en $\mathrm{kg} / \mathrm{m}^{3}$ y $\Delta U$ en $\mathrm{m} / \mathrm{s}$, obteniendo

$$
D_{0.5}=\frac{20 V_{L}^{0.5} \dot{m}_{L}^{0.1}\left(1+\dot{m}_{L} / \dot{m}_{A}\right)^{0.5} h^{0.1} \sigma^{0.2}}{\rho_{A T}^{0.3} U_{R}} .
$$

Mientras que en el trabajo [19] hizo similar transformación, tomando $D_{0.5}$ en $\mu \mathrm{m}, V$ en $\mathrm{m}^{2} / \mathrm{s}, W$ y $A$ en $\mathrm{kg} / \mathrm{s}, h$ en mm, $\sigma$ en $\mathrm{N} / \mathrm{m}, \rho_{A T}$ en $\mathrm{kg} / \mathrm{m}^{3}$ y $\Delta U$ en $\mathrm{m} / \mathrm{s}$, obteniendo

$d_{m}=0.004 V^{.5} W^{0.1}\left(1+\frac{W}{A}\right)^{0.5} h^{0.1} \sigma^{0.2} \rho_{a}^{-0.3} \Delta u^{-1}$.

La relación entre el diámetro de la masa $D_{0.5}$ y el diámetro medio Sauter (SMD) [25] es dada por $D_{0.53} / D_{32} \approx 1.2$.

\section{Resultados}

El presente análisis está basado en el trabajo [13] que utilizó aire como gas de atomización, Diesel como combustible cuya densidad es $830.2 \mathrm{~kg} / \mathrm{m}^{3}$, con una viscosidad dinámica de $2.4 \times 10^{-3}$, y la tensión superficial de $2.6 \times 10^{-3} \mathrm{~N} / \mathrm{m}$ y un inyector tipo Y; con diámetro para gas y combustible igual a $1 \mathrm{~mm}$ y el diámetro de la cámara igual a $1.8 \mathrm{~mm}$. Fueron efectuados los cálculos para determinar el diámetro medio de las gotas, mostrándose los resultados en la Fig.4. En esta figura también se presenta los resultados obtenidos al utilizar la ecuación (25). La ecuación
25 produce resultados muy pequeños, por lo cual no fueron incluidos en los gráficos para fines de comparación. La diferencia que existe entre los resultados obtenidos por las ecuaciones (23), (24) y (25) se debe a la posibilidad de que la cuestión no pase por una simple transformación de unidades, sino por un análisis dimensional de las propiedades que afectan el diámetro medio de las gotas. El recálculo para el coeficiente que debería tener la ecuación (24) arroja el valor 5.0237 .
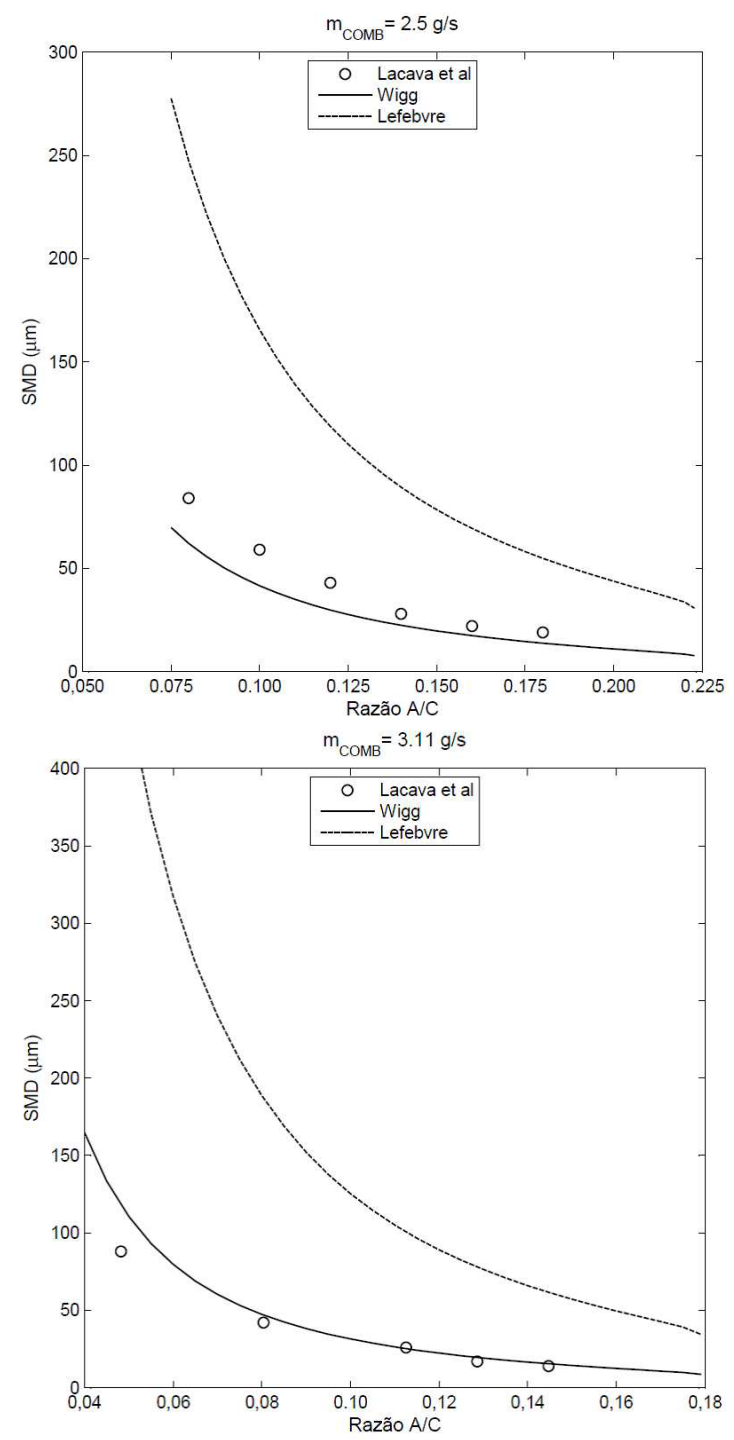

Figura 4: SMD calculado por las ecuaciones (23) y (24) para dos gastos másicos de combustible. 


\section{Conclusiones}

Las condiciones críticas del gas en el proceso de la atomización a la salida de la tobera del inyector sólo conducen a obtener el menor diámetro de gotas. Para cualquier otro caso, el flujo será subsónico con gasto másico menor que el crítico y con diámetro de las gotas mayor que el mínimo posible. Sin embargo, se pueden aceptar como satisfactorios valores de $80 \mu \mathrm{m}$ para una buena quema de combustible.

La presión crítica no debe ser calculada en base a la presión fuera del inyector, sino en base de la presión en la cámara de la mezcla, que está afectada por la presión con la cual llega el combustible a esta cámara. La caída de la presión a través del inyector está asociado a las pérdidas por expansión brusca, así como a los fenómenos de quiebra del chorro en gotas y la fricción con las paredes de la cámara.

Las pérdidas por expansión brusca, a la salida de la tobera del inyector obedecen a una ley cuadrática, tal como de predice en los tratados de mecánica de fluidos.

Un inyector puede trabajar en régimen subsónico si el gas de atomización llega a la cámara de mezcla con presión mayor que la presión crítica, pero esta presión no puede ser menor que la del medio circundante. Para las condiciones críticas, el gasto másico de gas será máximo y un incremento posterior de este gasto es imposible.

Las ecuaciones (24) y (25), son referenciadas en muchos trabajos y ofrecen resultados diferentes a la ecuación original de Wigg, (23). En la ecuación (24) se debe corregir el valor de la constante igual 20 por 5.0237. La diferencia entre los valores de estas ecuaciones puede ser posible a que la cuestión no pase por una transformación de unidades, si no por un análisis dimensional de las propiedades que afectan el diámetro de gotas.

\section{Referencias}

[1] H. Liu, Science and engineering of droplets: fundamentals and applications, William Andrew $\mathrm{Pu}-$ blishing, New York (1981).

[2] P. Andreussi, L. Tognotti, M. Graziadio y G. de Michelle; Atomization of Coal-Water Fuels by Pneumatic Nozzle: Characteristics of Spray, Aereosol Science and Technology 1(13), 35 (1990).

[3] P. Andreussi, L. Tognotti, G. de Michelle y M. Graziadio; Design and Characterization of TwinFluid Y-jet Atomizers, Atomization and Sprays 2, 45 (1992).

[4] H. Song y S. Y. Lee; Study of Atomization Mechanism of a Gas/Liquid Mixtures Flowing Through $Y$-Jet Atomizers, Atomizattion and Sprays 6, 193 (1996).

[5] H. S. Couto y D. Bastos-Netto; Generalized Liquid Film Atomization Theory, Journal of Thermal Science 9(3), 265 (2000).

[6] L. J. Guo, G. J. Li, B. Chen, X. J. Chen, D. D. Papailiou y Th. Panidis; Study on Gas-Liquid Two Phase Spraying Characteristics of Nozzles for the Humidification of Smoke, Experimental Thermal and Fluid Science 26, 715 (2002).

[7] Y. Song y M. Zhang; Mathematical Modeling and Experimental Verification of Interior Gas-Liquid Flows and Outflow Atomization Process for $Y$-jet Nozzles, Atomization and Sprays 14, 437 (2004).

[8] F. Barreras, A. Lozano, J. Barroso y E. Lincheta; Experimental Characterization of Industrial Twin-Fluid Atomizers, Atomization and Sprays 16, 127 (2006).

[9] G. Ferreira, F. Barreras, A. Lozano y J. A. García; Effect of the Inner Two-Phase Flow on the Performance of an Industrial Twin-Fluid Nozzle with an Internal Mixing Chamber, Atomization and Sprays 19, 873 (2009).

[10] H. Almeida, J. M. M. Sousa y M. Costa; Effect of the Liquid Injection Angle of the Liquid Jets in Subsonic Crossflow, $23^{\text {rd }}$ Annual Conference on Liquid Atomization and Spray Systems (ILASS), Brno, Czech Republic, September (2010).

[11] Y. Zhou, M. Zhang, J. Yu, X. Zhu y J. Peng; Experimental investigation and model improvement on the atomization performance of single-hole $Y$ jet nozzle with high liquid flow rate, Powder Technology № 199, 248 (2010).

[12] P. Mullinger y N. A. Chigier; The Design and Performance of Internal Mixing Multijet Twin 
Fluid Atomizers, Journal of the Institute of Fuel 47(393), 251 (1974).

[13] P. T. Lacava, J. A. Carvalho Jr, y M. Q. McQuay; Metodología para el diseño de atomizadores tipo $Y$, Información Tecnológica 9(6), Santiago de Chile, (1998).

[14] H. Clare y A. Radcliffe; An Airblast Atomizer for Use with Viscous Fuels, Journal of the Institute of Fuel 27(165), 510 (1954).

[15] L. D. Wigg; Drop Size prediction for Twin Atomizers, Journal of the Institute of Fuel $\mathbf{3 7}(256)$, 500 (1964).

[16] S. Nukiyama e Y. Tanasawa; Experiments on the Atomization of Liquids in an Airstream, Transaction of the Japan Society of Mechanical Engineers 5(18), 62 (1939).

[17] E. Mayer; Theory of Liquid Atomization in High Velocity Gas Streams, American Rocket Society Journal, 31, 1783 (1961).

[18] A. H. Lefebvre; Atomization and Sprays, CRC Press, Taylor \& Francis Group, New York (1989).
[19] N. A. Chigier; The Atomization and Burning of Liquid Fuel Sprays, Progress in Energy and Combustion Science 2, 97 (1976).

[20] P. K. Kundu y I. M. Cohen; Fluid Mechanics, $2^{\text {nd }}$ edition, Academic Press - Elsevier Science, New York (2002).

[21] R. B. Bird, W. B. Stewart y E. N. Lightfoot; Transport Phenomena, $2^{\text {nd }}$ edition, John Wiley \& Sons, New York (2001).

[22] M. J. Zucrow y J. D. Hoffman; Gas Dynamics, Vol. 1, John Wiley \& Sons, New York (1976).

[23] A. L. Pacífico; Análise teórico-experimental do escoamento bifásico no interior de bocais nebulizadores do tipo Y-Jet, Tese de Doutorado, Universidade de São Paulo, Brasil (2000).

[24] Crane Co. Engineering Division, Flow of fluid through valves, fittings and pipe, Crane Co., Technical paper $\mathrm{N}^{\circ} 410,11^{\mathrm{a}}$ edition (1969).

[25] H. Simons; The correlation Drop Size Distribution in Fuel Nozzles Spray, Journal of Engineering Power, 99, 309 (1977). 